\title{
Correction to: Soluble Epoxide Hydrolase Inhibition Attenuates Excitotoxicity Involving 14,15-Epoxyeicosatrienoic Acid-Mediated Astrocytic Survival and Plasticity to Preserve Glutamate Homeostasis
}

\begin{abstract}
Yi-Min Kuo ${ }^{1,2}$ • Pei-Chien Hsu ${ }^{1,3}$. Chia-Chi Hung ${ }^{1} \cdot$ Ya-Yu Hu ${ }^{1}$ Yu-Jie Huang ${ }^{1}$ Yu-Ling Gan ${ }^{1}$. Chun-Hua Lin ${ }^{4}$. Feng-Shiun Shie ${ }^{5} \cdot$ Wen-Kuei Chang ${ }^{2} \cdot$ Lung-Sen Kao $^{3,6} \cdot$ Mei-Yung Tsou $^{2} \cdot$ Yi-Hsuan Lee $^{1,3}$ (D)
\end{abstract}

Published online: 18 July 2019

(C) Springer Science+Business Media, LLC, part of Springer Nature 2019

\section{Correction to: Mol Neurobiol}

https://doi.org/10.1007/s12035-019-01669-8

The original version of this article unfortunately contained a mistake in Fig. 7d. The authors observed that the image of the GFAP/DAPI in the saline-treated wild type mice (WT Saline) was inadvertently rotated left 90 -degree by mistake. The corrected representative image is given below.

This correction does not change the values of quantification and the text of the figure legend, results, discussion, and conclusion. We apologize for any confusion caused to the readers.

The online version of the original article can be found at https://doi.org/ $10.1007 /$ s12035-019-01669-8

Yi-Hsuan Lee

yhlee3@ym.edu.tw

Yi-Min Kuo

ymkuo@vghtpe.gov.tw

Pei-Chien Hsu

pch99@ym.edu.tw

Chia-Chi Hung

chiachi@ym.edu.tw

$\mathrm{Ya}-\mathrm{Yu} \mathrm{Hu}$

s0963430980@gmail.com

Yu-Jie Huang

yjhuang118@ym.edu.tw

Yu-Ling Gan

yuling513@ym.edu.tw

Chun-Hua Lin

chlin@ukn.edu.tw

Feng-Shiun Shie

fshie@nhri.org.tw
Wen-Kuei Chang

wkchang@vghtpe.gov.tw

Lung-Sen Kao

1skao@ym.edu.tw

Mei-Yung Tsou

mytsou@vghtpe.gov.tw

1 Department and Institute of Physiology, National Yang-Ming University, 155 Sec. 2, Linong Street, Taipei 11221, Taiwan

2 Department of Anesthesiology, Taipei Veterans General Hospital and National Yang-Ming University School of Medicine, Taipei, Taiwan

3 Brain Research Center, National Yang-Ming University, Taipei, Taiwan

4 Department of Nursing, Kang-Ning University, Taipei, Taiwan

5 Center for Neuropsychiatric Research, National Health Research Institutes, Miaoli County, Taiwan

6 Department of Life Sciences and Institute of Genomic Sciences, National Yang-Ming University, Taipei, Taiwan 


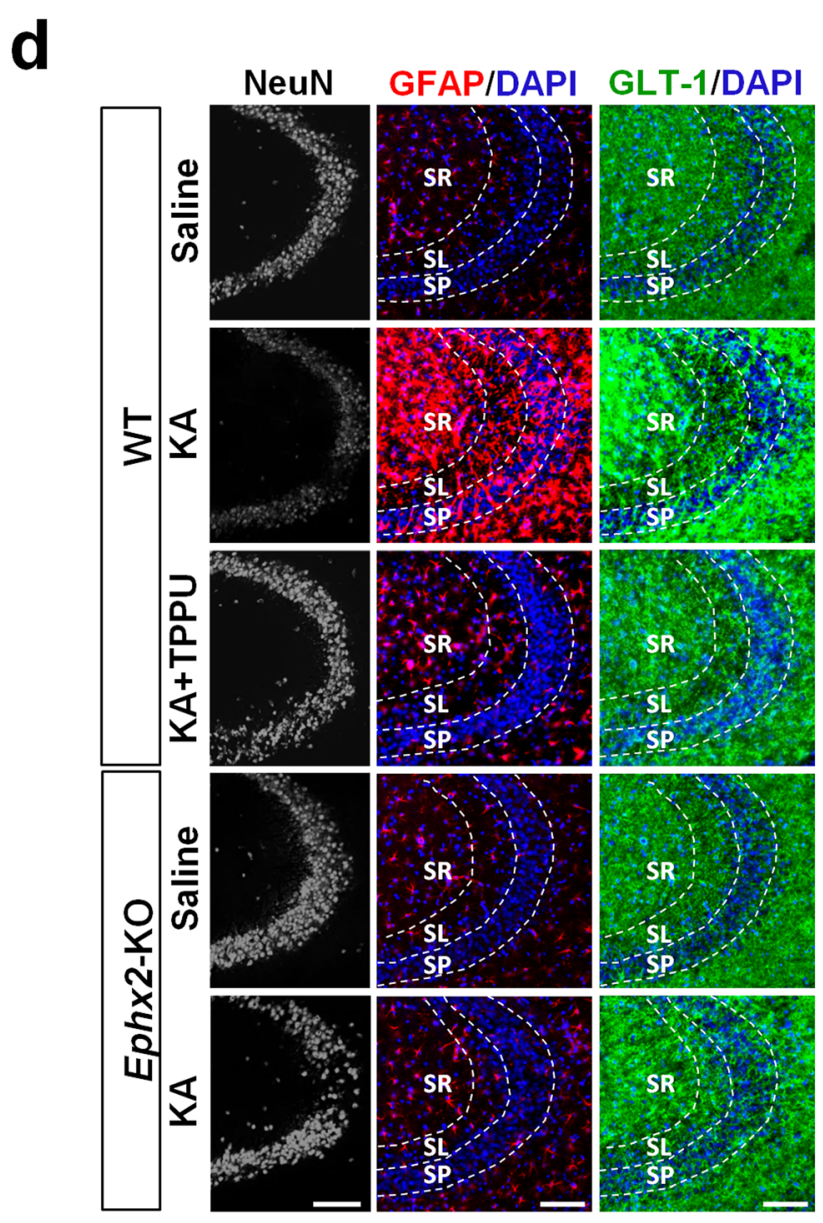

Kuo et al., Fig. 7d

Publisher's Note Springer Nature remains neutral with regard to jurisdictional claims in published maps and institutional affiliations. 